\section{Pegaptanib sodium for occult choroidal neovascularization in neovascular age-related macular degeneration: a prospective case series}

K Atmani, F Coscas, G Coscas and G Soubrane
Department of

Ophthalmology of Créteil, University Paris XII, Créteil France

Correspondence:

G Soubrane,

Department of

Ophthalmology,

University Paris XII,

Centre Hospitalier

Intercommunal de Créteil, 40 Avenue de Verdun,

Créteil

94010,

France

Tel: + 331451752 21;

Fax: + 33145175227 .

E-mail: gisele.soubrane@

chicreteil.fr

Received: 3 March 2008 Accepted in revised form: 29 May 2008

Published online: 18 July 2008

\section{Abstract}

Purpose To assess the effects of pegaptanib in the treatment of subfoveal occult choroidal neovascularisation (CNV) associated with neovascular age-related macular degeneration (NV-AMD) in a compassionate use program in France.

Methods Pegaptanib was authorized for patients with $\mathrm{CNV}$-associated visual impairment and in whom usual care (thermal laser photocoagulation or photodynamic therapy with verteporfin) was not appropriate. Patients with occult CNV lesions received intravitreous pegaptanib ( $0.3 \mathrm{mg}$ every 6 weeks) and were followed with repeated fluorescein angiography, scanning laser ophthalmoscopy-infracyanine green angiography, and ocular coherence tomography through 52 weeks.

Results Of 56 patients (predominantly occult, $N=22$; purely occult, $N=8$; occult with chorioretinal anastomosis, $N=12$; occult with pigment epithelial detachment, $N=14$ ), $30 \%$ had earlier treatment. All received eight pegaptanib injections. At week $52,79 \%$ were responders (lost $<15$ letters of visual acuity), $43 \%$ gained $\geqslant 0$ letters, and $9 \%$ gained $\geqslant 15$ letters. The best functional results were obtained in the predominantly and pure occult subgroups (responders, 86 and $75 \%$; gained $\geqslant 0$ letters, 50 and $\mathbf{5 0 \%}$ ). Maximum visual outcomes that correlated with morphologic improvements on each diagnostic imaging tool were seen after at least three injections. No significant ocular or systemic adverse events occurred.
Conclusion Treatment with pegaptanib was associated with objective functional improvements that can be correlated with objective clinical improvements on routine diagnostic imaging tools in patients with occult NV-AMD. Optimum treatment results appear after at least 4 months of therapy in the majority of cases.

Eye (2009) 23, 1150-1154; doi:10.1038/eye.2008.194; published online 18 July 2008

Keywords: age-related macular degeneration; choroidal neovascularisation; pegaptanib

Introduction

Age-related macular degeneration (AMD) is the leading cause of vision loss in the industrialized world ${ }^{1}$ with approximately $90 \%$ of severe vision loss attributable to the neovascular (NV) form. ${ }^{2}$ Until recently, treatment options for NV-AMD have been limited to either thermal laser photocoagulation or photodynamic therapy (PDT) with verteporfin (Visudyne, Novartis Pharmaceuticals Corporation), both of which are known to damage the underlying retinal and choroidal tissues in the treated area. ${ }^{3}$ The Vascular Endothelial Growth Factor Inhibition Study in Ocular Neovascularization (VISION) trial demonstrated the safety and efficacy of pegaptanib sodium (Macugen, Pfizer Inc.), a selective vascular endothelial growth factor (VEGF) inhibitor in eyes with NV-AMD. ${ }^{4}$ In this trial, pegaptanib was shown to stabilize vision in eyes with NV-AMD regardless of lesion size, angiographic subtype, or baseline visual acuity (VA). ${ }^{4}$ Earlier to the approval of pegaptanib by 
the European Medicines Agency (EMEA), patients in France with NV-AMD were eligible for treatment with pegaptanib through an authorization of temporary use (ATU) program of the French Health Authority. A study of subjects involved in this program was designed to evaluate the functional and morphologic effects of pegaptanib at different time points after treatment. In this report we describe our experience of close posttreatment follow-up of NV-AMD eyes with subfoveal occult choroidal neovascularization $(\mathrm{CNV})$ at the Department of Ophthalmology at the University Eye Clinic of Créteil in Paris, France.

\section{Methods}

This was a prospective study in which patients with NV$\mathrm{AMD}$, under a compassionate use program were eligible for treatment with pegaptanib if there was visual impairment in the second eye and treatment with usual care (observation, thermal laser photocoagulation, or PDT with verteporfin) was considered to be inappropriate or contraindicated. Although all subtypes of NV-AMD were eligible for treatment, only those with active subfoveal occult CNV lesions were included in this study. Eligibility criteria included patient age $\geqslant 50$ years, best-corrected VA of $20 / 40$ to $20 / 320$, lesion size $\leqslant 12$ disk areas, and intraocular pressure $\leqslant 23 \mathrm{mmHg}$. Patients with $\mathrm{CNV}$ owing to other causes were ineligible to participate in the study. Other exclusion criteria were treatment with PDT with verteporfin within 13 weeks preceding the baseline study visit, acute ocular or periocular infection, diabetic retinopathy, and severe cardiopathy.

All patients received pegaptanib $(0.3 \mathrm{mg})$ intravitreally every 6 weeks, with eight injections administered between day 0 and week 52 . The injection technique was consistent with that used in the VISION trial. ${ }^{2}$ Patients were examined before each injection and after each of the first three injections at days 8 (1 week), 21 (3 weeks), and 42 (6 weeks). After the fourth injection, patients were examined only on days 21 and 42 post-injection. At each examination patients were evaluated with a complete ophthalmic assessment, including VA, color fundus photography, fluorescein angiography (FA), scanning laser ophthalmoscopy-infracyanine green angiography (SLO-ICG-A), except for the day 8 visit, and optical coherence tomography (OCT; lines 3-5-10 mm and mapping), and for adverse events. We certify that all applicable institutional and governmental regulations concerning the ethical use of human volunteers were followed during this research.

\section{Results}

A total of 56 patients (39 females and 17 males) with a mean age of 78.2 years (62-92 years) were enrolled; all had active subfoveal occult CNV lesions with or without an associated clinical retinal pigment epithelial detachment (PED) or chorioretinal anastomosis (CRA; also called retinal angiomatous proliferation (RAP) lesion). Of the 56 eyes examined, there were 22 with predominantly occult (or minimally classic) CNV lesions, eight with pure occult CNV lesions, 12 with occult $\mathrm{CNV}$ associated with CRA and 14 with occult CNV associated with retinal PED. Seventeen patients $(30 \%)$ had received at least one earlier treatment with PDT with verteporfin in the study eye given 13 or more weeks before enrollment.

\section{Functional outcomes}

After eight injections, 44 (79\%) of the eyes examined were responders (loss of $<15$ letters of VA); 24 (43\%) were maintainers (maintained or gained $\geqslant 0$ letters) and five $(9 \%)$ were gainers (gained $\geqslant 15$ letters) (Figure 1$)$. The proportions of responders, maintainers, and gainers were generally similar across the four occult lesion subgroups. Given the small sample sizes, no correlation can be inferred between functional outcomes, including the five gainers, and the precocity of the lesions; most patients were treated after a waiting period of many weeks, before receiving authorization to proceed. The best VA outcomes were obtained for eyes with predominantly occult (minimally classic) CNV lesions with $86 \%$ of eyes being responders at 52 weeks and $50 \%$ maintaining or gaining VA. Those with pure occult CNV lesions were also stabilized, with $75 \%$ of eyes being responders and $50 \%$ with either unchanged or increased VA. For occult CNV lesions associated with CRA and PED, representing more severe forms of NV-AMD, 75 and $71 \%$ of eyes, respectively, were responders at 52 weeks (Figure 2).

At 52 weeks, the mean VA across all eyes with occult CNV lesions was stable, slightly decreasing from 20/80

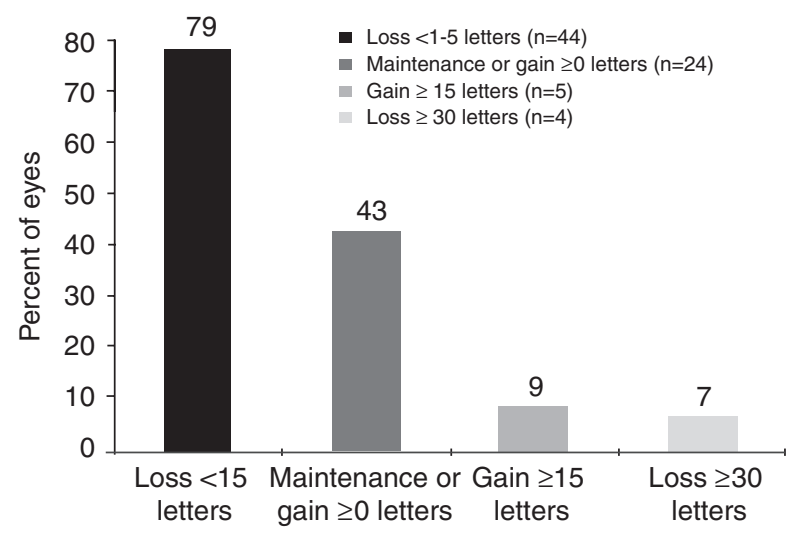

Figure 1 Overall response rates at week 52 for pegaptanibtreated eyes with occult choroidal neovascularisation (56 eyes). 
at baseline to 20/100. In eyes with minimally classic $\mathrm{CNV}$ the mean VA was unchanged from baseline to 52 weeks although there was a decrease in mean VA in the other subgroups (Table 1). No significant ocular or systemic adverse events occurred during the study.

\section{Morphologic outcomes}

Color fundus photography, FA, SLO-ICG-A and OCT were used to evaluate the morphologic changes associated with the use of pegaptanib for the treatment of occult NV-AMD lesions and were correlated with functional results. In eyes with minimally classic CNV, there was a decrease in the size and leakage of the classic component after the third injection in 18 of 22 (82\%) eyes and stabilization in the lesion size in nine of $22(41 \%)$ eyes on FA. SLO-ICG-A demonstrated no progression (5 eyes), regression of the perfused NV network present in the early phase in two of seven eyes and showed no change in the size of the late-phase plaque in 8 of 22 eyes. During follow-up, OCT imaging showed a rapid decrease in intra-retinal and sub-retinal fluid; this was observed at the first post-injection visit (day 8) in 13 of 22 (59\%) eyes. For all eyes with minimally classic CNV lesions, the mean decrease in central retinal thickness measured by OCT at 52 weeks was $102 \mu \mathrm{m}$ (from a

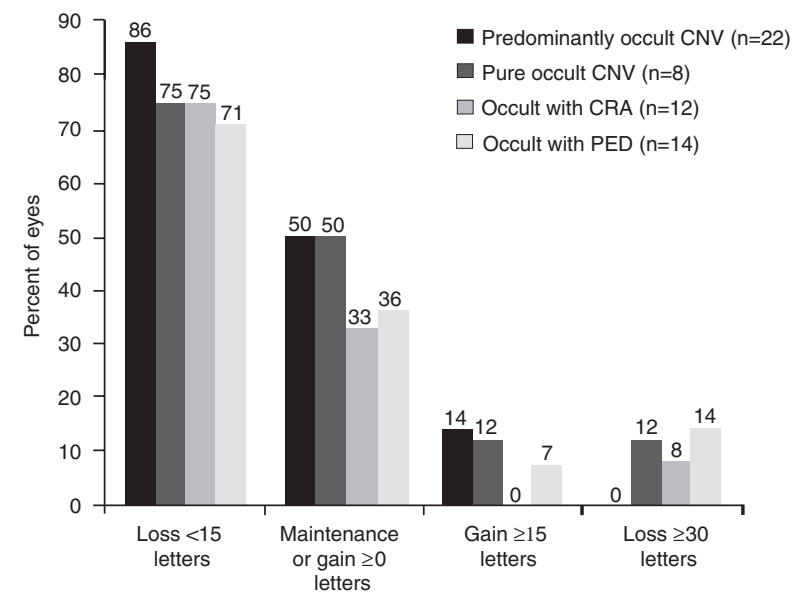

Figure 2 Response rates at week 52 across occult choroidal neovascularisation lesion subgroups in pegaptanib-treated eyes. baseline of 364 to $262 \mu \mathrm{m})$. At the end of the treatment in the responder eyes, the classic component of the CNV was often not discernible on OCT imaging; the hyperreflective fusiform neoformation corresponding to the pre-epithelial part of the lesion regressed or disappeared in 14 of $22(64 \%)$ eyes, as did exudative signs. At the same time, indirect signs of occult neovascularization, such as elevation, irregularity, or splintering of the RPE remained unchanged.

When combining the results of the various imaging examinations performed on responder eyes with predominantly occult CNV lesions, the reduction in fluorescein leakage originating from the classic component of the lesion on FA generally coincided with the resorption of intra-retinal fluid on OCT. This fluid reduction was in parallel with the resolution of the patient's complaint of metamorphopsia. The improvements in VA occurred slowly and were not evident until after at least three injections. Interestingly, when improvements in VA occurred, improvements in near vision preceded improvement in distance vision in all cases, often by several weeks. In more than half of the cases a mild increase in lesion size, measured by FA and SLO-ICG-A, was observed following treatment without any corresponding detriment to the patients' VA. The mean VA remained unchanged after 1-year follow-up within this subgroup, having stabilized at 20/100. For responder eyes, regression of lesions under treatment, especially the pre-epithelial part, involved atrophy more than fibrosis, based on biomicroscopy and imaging aspects.

In the eight patients with pure occult CNV lesions, the morphologic changes following treatment with pegaptanib were less obvious. By FA, the appearance of many lesions was unchanged although in some cases a moderate increase in fluorescein diffusion and lesion size was noticed. SLO-ICG-A proved more useful than FA in defining the limits of the lesion by highlighting the NV network and demonstrating the area of the late-phase plaque. With OCT imaging, the resorption of the intra-retinal and sub-retinal fluid associated with pure occult $\mathrm{CNV}$ lesions was slower than that observed in the predominantly occult CNV lesions. The other signs, such as RPE elevation, were not affected by treatment.

Table 1 Mean visual acuity (VA) at baseline and 52 weeks

\begin{tabular}{lccccc}
\hline & $\begin{array}{c}\text { Total occult } \\
\text { CNV } \\
(56 \text { eyes })\end{array}$ & $\begin{array}{c}\text { Predominantly occult } \\
\text { (minimally classic) } \\
\text { CNV (22 eyes) }\end{array}$ & $\begin{array}{c}\text { Pure occult } \\
\text { (occult only) } \\
\text { CNV (8 eyes) }\end{array}$ & $\begin{array}{c}\text { Occult CNV } \\
\text { with CRA } \\
\text { (12 eyes) }\end{array}$ & $\begin{array}{c}\text { Occult CNV with } \\
\text { PED (14 eyes) }\end{array}$ \\
\hline Mean baseline VA & $20 / 80$ & $20 / 100$ & $20 / 80$ & $20 / 64$ & $20 / 64$ \\
Mean VA at 52 weeks & $20 / 100$ & $20 / 100$ & $20 / 125$ & $20 / 125$ & $20 / 100$ \\
\hline
\end{tabular}

$\mathrm{CNV}=$ choroidal neovascularisation; $\mathrm{CRA}=$ chorioretinal anastomosis; $\mathrm{PED}=$ pigment epithelial detachment; $\mathrm{VA}=$ visual activity. 
As noticed for those with minimally classic CNV, eyes with occult CNV experienced a gradual improvement in VA after several injections. Changes in VA paralleled the resolution of the intra-retinal fluid associated with the lesion on OCT; however, there was no correlation between increase in lesion size on SLO-ICG-A imaging and visual function. For all responder eyes in this subgroup, the lesions atrophied with little fibrosis, based on biomicroscopy and imaging.

In our experience, occult $\mathrm{CNV}$ lesions that are associated with CRA have a poor prognosis irrespective of the treatment. In the 12 eyes with CRA in this series, the response to pegaptanib was quite variable and difficult to characterize. FA often showed an increase in both the total size of the lesion and fluorescein leakage. FA identified development of a classic NV membrane around the anastomoses in two eyes with an associated severe loss of vision in one eye. SLO-ICG-A facilitated the diagnosis and visualization of these changes. The effects of pegaptanib on OCT varied; in some cases there was a moderate reduction in the quantity of intra-retinal fluid whereas in other cases there was an appearance or worsening of cystoid macular edema; the PEDs persisted after treatment in all cases.

Occult CNV associated with PED is considered to be a severe form of NV-AMD. In the 14 eyes with these lesions, FA, SLO-ICG-A, and OCT were each useful for demonstrating the progressive change in the PED following treatment with pegaptanib. In three eyes, hyperfluorescence of the PED demonstrated on FA persisted and became inhomogeneous on its border, indicating a progression from serous PED associated with CNV to fibrovascular PED. This CNV enlargement did not always lead to visual impairment. VA either stabilized or improved with collapse of the PED or with decreased retinal edema when the foveal depression was restored. In two other eyes, the PED resolved almost completely with moderate growth of CNV lesion and VA stabilization. The lesions and VA remained relatively stable in five eyes and worsened in two others, leading to severe loss of VA. An extrafoveal tear of the retinal pigment epithelium occurred in two eyes without loss of VA, because of the extrafoveal location of the rip. SLO-ICG-A imaged the change in the nature of the PED because of the contrast between the hypocyanescent detached pigment epithelium and the hypercyanescent occult neovascularization, whereas OCT allowed better visualization of the overall changes in the retinal architecture (organization of retinal layers, distribution of intra-retinal fluid), and PED content (changes of reflectivity).

Images of three representative cases shown before and after eight pegaptanib injections are posted as Supplementary Information on the journal's web site.

\section{Discussion}

Historically, patients with NV-AMD have had only laserbased treatment options, either thermal laser photocoagulation or PDT with verteporfin. As both of these therapies can cause iatrogenic damage to the retina and choroid in the process of treating neovascular lesions, there are limitations to the use of these treatments in all CNV lesion types and sizes. Clinical studies demonstrate that the benefit of PDT is primarily in eyes with predominantly classic CNV lesions, ${ }^{5}$ the angiographic subgroup for which PDT received regulatory approval and which occurs in 17-20\% of NVAMD patients. ${ }^{6-8}$ The lack of efficacy of PDT with verteporfin in patients with occult CNV lesions was recently confirmed in the Visudyne in Occult (VIO) trial. ${ }^{9}$ The VISION trial showed that medical treatment with a selective anti-VEGF therapy plays an important role in the management of all NV-AMD subtypes. To enhance the understanding of patients' morphologic changes occurring during the course of treatment with a selective VEGF inhibitor in relation with the functional response, this prospective observational study was designed for active subfoveal occult CNV eligible for treatment with pegaptanib under the protocol and criteria of the French Health Authority.

The visual outcomes at week 52 seen in our overall occult CNV lesion study population (79\% responders, $43 \%$ maintainers, and 9\% gainers) were somewhat better than those reported in the VISION trial, ${ }^{4}$ which analyzed globally all types of CNV lesions. As in the VISION trial, many patients in this study had long-standing disease and were subject to a treatment delay between the diagnosis of NV-AMD and authorization for treatment with pegaptanib in the compassionate use program. Such delays in treatment are known to be associated with vision losses that may impact the visual prognosis. A retrospective subgroup analysis of VISION trial patients with earlier-stage lesions suggest that treatment of early disease is likely to produce better outcomes with $20 \%$ of eyes with active occult disease gaining $\geqslant 3$ lines of vision. ${ }^{3}$ Similarly, small retrospective case series of patients treated with pegaptanib in the United States, after the drug was launched in January 2005, are consistent with the expectation that earlier treatment produces better clinical outcomes. ${ }^{10,11}$

In this study, treatment with pegaptanib was associated with objective functional improvements that could be correlated with clinical improvements on each of the diagnostic imaging tools routinely performed in the assessment of NV-AMD patients. In eyes with predominantly occult or minimally classic CNV and FA, the following treatment revealed a slowing in the growth of the total area of the lesion, CNV size, and mainly a 
decrease in severity of leakage in the majority of cases after three or four injections. Review of both early and late phases of the SLO-ICG-A is important as it demonstrates the exact extent of the response of the perfused early NV network to the selective anti-VEGF. A decrease in intra-retinal and sub-retinal fluid and a decrease in retinal thickness on OCT were rapid following each injection particularly in the predominantly and pure occult CNV lesions, whereas the changes of accumulated fluid in patients with PED and CRA were variable.

These results and observations show that pegaptanib stabilizes VA in patients with occult NV-AMD lesions regardless of their FA appearance. The maximum visual outcomes appear after at least three injections in the majority of cases. Of the four occult CNV lesion subgroups evaluated in this study the best functional results were obtained with predominantly and pure occult CNV with repeatable effects and quite similar responses. The results in the other subgroups were only slightly less and consistent with the recognition that treatments for occult CNV lesions associated with PED or CRA (or RAP) mighty show less efficacy as these lesions are considered to be severe forms of NV-AMD. These findings suggest that for optimum treatment results with pegaptanib, the duration of treatment must be at least 4 months and that multiple intravitreal injections at 6-week intervals are required.

In this study it was observed that pegaptanib seems to act more on the hyperpermeability component than on endothelial cell proliferation of the occult CNV lesions. Although the decrease and even disappearance of leakage is a hallmark of anti-VEGF treatment, the lack of a similar effect on the neo-vessel proliferation across the lesion subgroups is somewhat surprising. In responder eyes in the minimally classic and occult CNV lesion subgroups there was an absence of visibility of the NV and supporting fibrous tissues after treatment with all three means of imaging, suggesting atrophy of the NV tissue. What becomes of the NV tissue and why no fibrosis develops remain noteworthy questions as the precise elucidation of this mechanism could help to provide early clues to recognize patients most likely to be responders.

Now that pegaptanib is available by prescription, retinal specialists will be able to initiate treatment immediately after the diagnosis of NV-AMD. With greater awareness between ophthalmologists and other physicians for the identification and monitoring of at-risk patients, early diagnosis of NV-AMD should become more realistic. Although prospective data are needed to confirm the benefits of early treatment, it is reasonable to expect effective prevention of vision loss in patients diagnosed and treated earlier in the course of this common degenerative disease.

\section{Acknowledgements}

Editorial support, including contributing to the first draft of the manuscript, revising the paper on the basis of author feedback, and styling the paper for journal submission was provided by Gardiner Caldwell US with assistance from Zola Associates and funded by Pfizer Inc.

\section{References}

1 Resnikoff S, Pascolini D, Etya'ale D, Kocur I, Pararajasegaram R, Pokharel GP et al. Global data on visual impairment in the year 2002. Bull World Health Organ 2004; 82: 844-851.

2 Ferris III FL, Fine SL, Hyman L. Age-related macular degeneration and blindness due to neovascular maculopathy. Arch Ophthalmol 1984; 102: 1640-1642.

3 Gonzales CR, for the VEGF Inhibition Study in Ocular Neovascularisation (VISION) Clinical Trial Group. Enhanced efficacy associated with early treatment of neovascular age-related macular degeneration with pegaptanib sodium: an exploratory analysis. Retina 2005; 25 : 815-827.

4 Gragoudas ES, Adamis AP, Cunningham Jr ET, Feinsod M, Guyer DR. Pegaptanib for neovascular age-related macular degeneration. N Engl J Med 2004; 351: 2805-2816.

5 TAP Study Group. Photodynamic therapy of subfoveal choroidal neovascularization in age-related macular degeneration with verteporfin: one-year results of 2 randomized clinical trials - TAP report. Treatment of agerelated macular degeneration with photodynamic therapy (TAP) Study Group. Arch Ophthalmol 1999; 117: 1329-1345.

6 Margherio RR, Margherio AR, DeSantis ME. Laser treatments with verteporfin therapy and its potential impact on retinal practices. Retina 2000; 20: 325-330.

7 Bermig J, Tylla H, Jochmann C, Nestler A, Wolf S. Angiographic findings in patients with exudative agerelated macular degeneration. Graefes Arch Clin Exp Ophthalmol 2002; 240: 169-175.

8 Olsen TW, Feng X, Kasper TJ, Rath PP, Steuer ER. Fluorescein angiographic lesion type frequency in neovascular age-related macular degeneration. Ophthalmology 2004; 111: 250-255.

9 QLT Inc. QLT announces two year results of Visudyne in Occult (VIO) Trial; September 20 2005b. http:// www.qltinc.com/Qltinc/pages/pagespressdetail. cfm?PressID $=252 \&$ YearRange $=2005 \&$ InternetPageID = 31.Accessed January 29, 2007.

10 Lupovitch J, Quiram PA, Hassan TS, Williams GA. Macugen as primary therapy for AMD lesions of all angiographic subtypes [abstract]. Invest Ophthalmol Vis Sci 2006; 47: E Abstract 5217.

11 Kirsch LS, Hairston RJ. Macugen in the treatment of exudative age-related macular degeneration: Experience at the Eye Institute of West Florida. Paper presented at Euretina 2006; May 18-21 Lisbon, Portugal, 2006.

Supplementary Information accompanies the paper on Eye website (http://www.nature.com/eye) 